\title{
Uma Proposta de Barramento de Dados para Integração de Serviços Públicos Digitais
}

\author{
Kelson Almeida ${ }^{1}$, Rostand Costa ${ }^{1}$ \\ ${ }^{1}$ Centro de Informática - Universidade Federal da Paraíba (UFPB) \\ Código Postal 58.058-600 - João Pessoa - PB - Brazil \\ kelson.victorddcx.ufpb.br, rostand@lavid.ufpb.br
}

\begin{abstract}
The use of technologies that aim to reduce bureaucracy in public services is important for societies around the world. With the use of artifacts and technological practices, it is possible to save financially and work time. The interoperability of data and information between different Information Systems in any modern technology ecosystem is a necessary item for queries and validations in different databases. This exchange of data in government environments is necessary in the fast and effective delivery of digital public services to the population. This work proposes the use of a bus that helps interoperability aiming at a technological boost in Electronic Governments. This bus proposal, called IO Data Bus, proved to be promising through practical simulations using the data exchange bus X-Road in the proof of concept, an element that provided the technological base of Estonia Digital. As a basis for our experiment, we used the the payment of Emergency Aid in Brazil example, during the crisis caused by the pandemic caused by the new coronavirus.
\end{abstract}

Resumo. $O$ uso de tecnologias que visam desburocratizar serviços públicos são de suma importância por sociedades ao redor de todo o mundo. Com a utilização de artefatos e práticas tecnológicas, é possível economizar financeiramente e em tempo de trabalho. A interoperabilidade de dados e informações entre diferentes Sistemas de Informação em qualquer ecossistema moderno de tecnologia é um item necessário para consultas e validações em bases de dados distintas. Essa troca de dados em ambientes governamentais se faz necessária na entrega rápida e eficaz de serviços públicos digitais para a população. Este trabalho propõe a utilização de um barramento que auxilia a interoperabilidade visando um impulso tecnológico em Governos Eletrônicos. Tal proposta de barramento, chamado IO Data Bus, se mostrou promissor através de simulações práticas utilizando na prova de conceito o barramento de troca de dados $X$ Road, elemento esse que proporcionou a base tecnológica da Estônia Digital. Como embasamento no nosso experimento, utilizamos o exemplo do pagamento do Auxílio Emergencial no Brasil, durante o período de crise causada pela pandemia do novo coronavírus.

\section{Introdução}

No Brasil, a prestação de serviços públicos é dever da Administração Pública, tido por alguns doutrinadores como a principal justificativa e finalidade do Estado [da Purificação 2019]. Consiste em prestações materiais das mais diversas, todas promotoras de benefícios para os administrados, benfazejos individuais e coletivos. 
Sistemas de Computação e artefatos tecnológicos já se tornaram itens indispensáveis na busca pela diminuição de tarefas burocráticas e economia em esforço e tempo de trabalho entre pessoas, empresas e governos ao redor do mundo. Esses Sistemas de Informação, na maioria dos casos, precisam trocar dados entre si para a realização das tarefas necessárias.

Ações que tem como objetivo a troca de dados entre diferentes entidades tecnológicas de maneira segura, rápida e íntegra. Essas características são fatores que alavancam o processo de digitalização de serviços públicos, reduzindo assim o tempo de resposta em atendimentos e solicitações da sociedade e público em geral, além do mais pode fornecer mais transparência e padronização.

Em grandes esferas governamentais, a interoperabilidade entre diferentes Sistemas de Informação, onde, geralmente, há uma enorme quantidade de requisições e armazenamento de informações. Um dos grandes desafios para a prestação e fornecimento de serviços públicos de maneira digital é a interoperação entre diferentes entidades que necessitam realizar troca de dados. Segundo [dos Santos 2011], os Governos Eletrônicos devem se empenhar para alcançar a interoperabilidade entre os seus serviços digitais, como razões são ditos a melhora na tomada de decisão, melhor coordenação das ações governamentais para a oferta de serviços à população, a interoperabilidade ser a base na oferta de serviços através de um único ponto de acesso e redução de custos, sistemas interoperáveis evitam a implantação de novos sistemas que anteriormente se fariam necessários e ainda aumentam o leque de alternativas e aquisições de soluções, evitando a dependência de fornecedores únicos.

Em [Brizzi Neto 2007] é levantado o fato, de que, de acordo com o Banco Mundial, o Brasil é o país mais burocrático do mundo. Práticas antigas e engessadas ainda são postas como forma de prestação de serviços no país. Ainda de acordo com os autores, empresas brasileiras gastam quase duas mil horas e 60 bilhões de reais apenas em burocracia tributária todos os anos.

Em contraponto, pequenos países europeus como a Estônia e Finlândia são exemplos em digitalização e desburocratização dos seus serviços públicos, onde esses são prestados quase em $100 \%$ de maneira digital e interoperável, utilizando como suporte uma identificação única para cada cidadão ou empresa e uma camada de troca de dados chamada X-Road. Desde a adoção dessa camada no ano de 2001, ela é a responsável pela interconexão altamente madura, segura e escalável das instituições estonianas para os setores públicos e também privados [Saputro et al. 2020] [Kalja 2011].

Com isso, não somente chegar-se a artefatos tecnológicos, como também obter meios que facilitem a interoperabilidade entre sistemas governamentais são vistos como itens fundamentais para atender situações cotidianas de Governo Eletrônico e diminuir a grande burocracia existente, principalmente, em países emergentes como o Brasil. Neste trabalho, é proposto o IO Data Bus, um barramento de troca de dados que busca auxiliar na interoperabilidade em Governo Eletrônico, fazendo assim que diferentes sistemas interajam em alinhamento de propósitos.

O restante do artigo está organizado da seguinte maneira. A Seção 2 traz alguns trabalhos presentes na literatura que possuem correlação com a pesquisa. A Seção 3 apresenta o barramento proposto, sua arquitetura e implementação. A Seção 4 detalha o 
nosso planejamento experimental e prova de conceito. A Seção 5 apresenta os resultados e análise da pesquisa e por fim a Seção 6 traz a conclusão do trabalho.

\section{Trabalhos Relacionados}

A impulsão da digitalização na entrega de serviços para a sociedade em esferas governamentais vem se fazendo como fator necessário ao decorrer dos anos. Em 2004 foi publicado o The European Interoperability Framework for Pan-European E-Goverment Services [Framework 2004]. O surgimento desse guia teve como objetivo definir um conjunto de recomendações para serviços de Governo Eletrônico em que administrações públicas, empresas e cidadãos pudessem interagir além fronteiras no contexto pan-Europeu. Em [Srivastava and Teo 2004], os autores trouxeram um outro framework de transformação em Governo Eletrônico. Esse guia explorou essa transformação através de facilitadores e marcos nomeados. A utilização de guias de boas práticas em e-gov também é utilizada no contexto brasileiro, com o e-PING, ou Padrões de Interoperabilidade do Governo Eletrônico [Brasileiro 2018]. Antes da aplicação de qualquer prática tecnológica no barramento realizamos um estudo das práticas utilizadas no guia.

$\mathrm{Na}$ aliança com essas as boas práticas, se fez necessário enxergar artefatos tecnológicos que dessem um melhor suporte aos modelos de e-gov. Segundo [Jaeger and Thompson 2003], o principal propósito na busca de soluções de interoperabilidade no setor público é a habilitação da troca de dados dentro da organização pela internet. Também é importante conscientizar pessoas entre os cidadãos e funcionários do governo. Isso oferece vantagens na direção a um governo eletrônico. Essa utilização de um artefato tecnológica está presente na proposta do nosso trabalho visando um melhor fluxo interoperável.

Em [Nielsen 2017] são descritos cinco itens considerados como essenciais e prérequisitos de Governo Eletrônico e que ocasionaram, por exemplo, o sucesso das soluções estonianas no seu modelo de $e$-gov. Esses itens são a infraestrutura, o grau de instrução digital da população, identificação única e digital, registros digitais e troca de dados formal. Dessa forma, apresentaremos uma proposta de Identificação Digital e Única para nossa prova de conceito.

Open-source e chave da digitalização de sucesso entre serviços públicos e privados são características que trouxeram e trazem com o $X$-Road vários benefícios na sua utilização. Argentina, Azerbaidjão, El Salvador, Ilhas Faroe, Finlândia, Islândia, Quirguistão, Namíbia e Palestina são exemplos de países, citados por [Saputro et al. 2020], que reconheceram os conceitos do barramento estoniano e implantaram soluções semelhantes para seus modelos de e-gov.

Através desse fato, pôde ser observado em [Saputro et al. 2020] a análise de prérequisitos na adoção do $X$-Road em um país, através de um estudo comparativo. Esses pré-requisitos foram validados através de entrevistas qualitativas e identificando alguns pré-requisitos adicionais. Servindo como base para a aplicação na nossa simulação de contexto de e-gov no governo brasileiro. Observando assim que somente a implantação de um barramento como $X$-Road, não significará o total benefício e sucesso de uma solução de governo digital, sendo necessário um conjunto itens como capacidade técnica, Politicas de Segurança da Informação, Identificação Única e outros aspectos culturais. 


\section{Proposta do Barramento: IO Data Bus}

A utilização de um barramento de troca de dados que dê suporte à uma melhor interoperabilidade vem trazendo sucesso à modelos de Governos Eletrônicos em países como Estônia e Finlândia. Observando a situação do Governo Eletrônico brasileiro no que diz respeito à interoperabilidade, até o presente momento, nosso país ainda não possui nenhuma solução tecnológica semelhante. Nesses países europeus citados, essa solução é chamada de $X$-Road. Há até uma proposta de modernização do $e$-gov brasileiro no âmbito federal [Digital 2020]. A ideia de unificar vários documentos de identificação dos cidadãos em um único, presente nessa proposta de impulso digital, foi e está sendo um dos fatores chave para o sucesso na integração dos chamados -Services, serviços públicos digitais, na Estônia. Com o apoio da camada de troca de dados, X-Road, a Estônia é o país mais digital na entrega de serviços públicos no mundo.

Analisando esses fatos, esse trabalho propõe o IO Data Bus ou Interoperability on Data Bus, do português Interoperabilidade no Barramento de Dados. Esse barramento tem como objetivo, aprimorar a conexão dos serviços públicos digitais brasileiros para a entrega de uma melhor troca de dados entre entidades governamentais e população, funcionando assim como intermediário dessa conexão.

Como nosso barramento propõe otimização em práticas interoperáveis e vamos focar no $e$-gov brasileiro, iremos utilizar algumas premissas presentes na arquitetura $e P I N G$, que são os Padrões de Interoperabilidade do Governo Eletrônico que está em vigor no âmbito governo federal (Poder Executivo) e que traz premissas da utilização de Governo Eletrônico no Brasil, como isso temos como objetivo fornecer um melhor embasamento às técnicas propostas e partir de soluções, ou guia de boas práticas, que já são empregadas no contexto brasileiro e que estão contidas nas especificações técnicas da segmentação do documento. [Brasileiro 2018].

Com isso, para uso na nossa proposta de barramento extraímos do segmentos de interconexão alguns componentes como protocolos de transferência de arquivo (HTTP e FTP), sincronismo de tempo (NTP), serviços de nomeação de domínio (DNS - gov.br e DNSec), protocolos de trasporte (TCP e UDP). Alguns segmentos de segurança como transferência de dados em redes inseguras (TLS), certificado digital (X.509 v3 - ICPBRASIL), carimbo de tempo (Time-Stamp Protocol). Na segmentação foi extraída a utilização da linguagem para intercâmbio de dados (JSON) e o protocolo para acesso a webservice (HTTP/1.1).

A priori, apenas alguns desses itens estarão presentes na nossa demonstração de planejamento experimental, esses segmentos foram extraídos de acordo com um estudo técnico da documentação do X-Road [NIIS 2020], com isso, a nível tecnológico essas características do guia serviram como referencial técnico para a utilização de alguns componentes e tecnologias no barramento.

Para a estratégia e política de dados da utilização do barramento, propomos o uso do certificado digital ICP Brasil, como citado anteriormente, extraído do ePING. Na ilustração da Figura 1 esse certificado será fornecido por uma possível entidade responsável pelo gerenciamento do IO Data Bus para o serviço e/ou entidade pública ou privada que participará do fluxo interoperável do barramento para ter acesso ao mesmo. De acordo com a necessidade do serviço digital em questão, podem ser implementadas 
APIs para a conversação entre os sistemas que trocam os dados e a intermediação do barramento. Na próxima seção os aspectos tecnológicos dessa política de troca de dados serão mais detalhados.

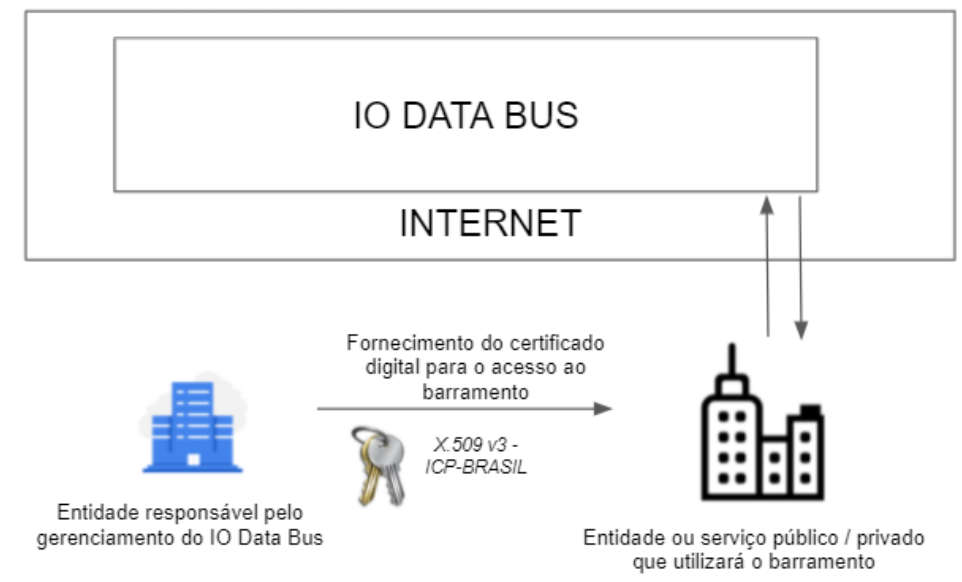

Figura 1. Estratégia de utilização do barramento

\subsection{Arquitetura Proposta}

A Figura 2 ilustra a estrutura básica do nosso barramento em um contexto genérico do Governo Eletrônico. A camada interoperável será responsável pelo gerenciamento da troca de dados entre os stakeholders do e-gov.

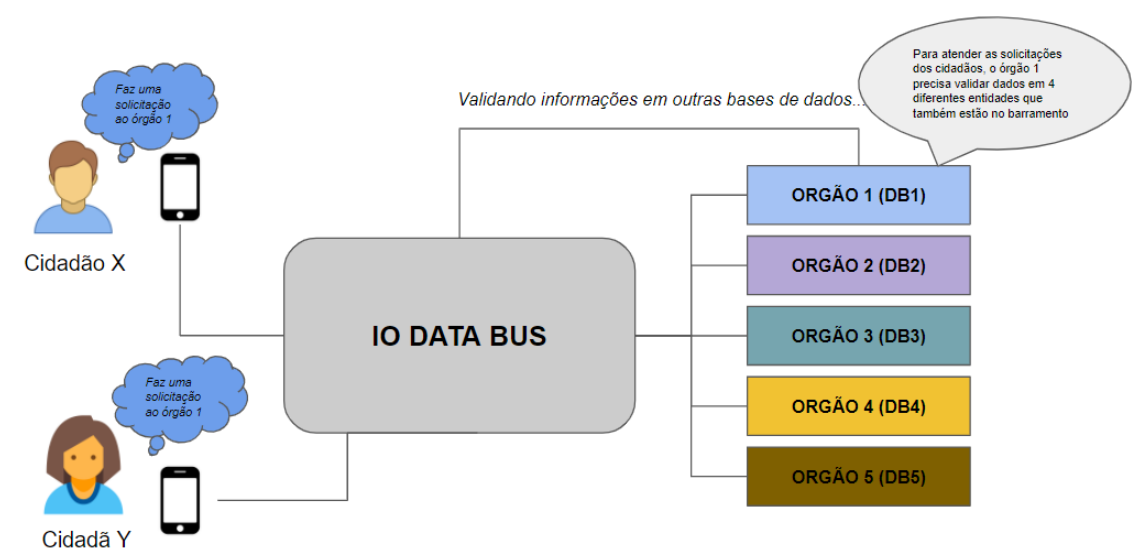

Figura 2. Estrutura básica do IO Data Bus no e-gov.

O trabalho é desenvolvido no planejamento e execução de uma implementação real que forneça um protótipo funcional do barramento. Os itens a seguir especificam algumas das características que fazem parte do sistema:

- Divisão em módulos: O barramento é dividido em módulos Cliente e Servidor, esses módulos irão possuir submódulos onde serão conectados todas as entidades participantes do Governo Eletrônico através do IO Data Bus. 
- Gravação de valores evidenciais $(\operatorname{logs})$ de transações com cópias em DLTs: Para garantir uma melhor segurança e imutabilidade de valor evidencial das transações ocorridas dentro do barramento, todos os registros de logs poderão ser armazenados em livro-razão distribuído, do inglês Distributed Ledger Technology, de acordo com a necessidade governamental ou próprio órgão presente no barramento.

- Segurança criptográfica entre entidades autorizadas no barramento: Os Sistemas de Informação trocam apenas mensagemns criptografadas por canal de segurança TLS. As entidades conectadas ao barramento necessitam de certificação digital de autenticação que estejam em conformidade com os certificados permitidos pelo barramento.

- Descentralização de banco de dados: Com diferentes sistemas interligados aos módulos clientes e servidores, não haverá ponto crítico de falha no âmbito geral do Governo Eletrônico. Várias bases de dados podem ser acessadas para atender diversas solicitações, tornando assim o ambiente muito mais interoperável.

- Padronização, Independência de Plataforma e Adaptabilidade: A troca de dados vai se dar pela arquitetura REST e os dados trafegados na camada estão no formato JSON. Dessa forma, o desenvolvimento de softwares não irão necessidade de implementações na mesma linguagem e/ou que operem na mesma plataforma. Os tipos de dados na interoperação estão no mesmo formato, ocorrendo a padronização, alcançando também independência e adaptabilidade das tecnologias e plataformas.

A Tabela 1 detalha os elementos presentes na arquitetura do barramento e que englobam o processo de interoperabilidade para o Governo Eletrônico. Para um melhor entendimento dessa arquitetura, a Figura 3 ilustra a seguinte suposição: um cidadão se dirige a um órgão X para buscar algum tipo de atendimento público, para atender a solicitação o Sistema de Informação do órgão $X$ precisa de dados externos que se encontram nos bancos de dados dos órgãos $\mathrm{Y}$ e $\mathrm{Z}$ conectados ao barramento IO Data Bus.

Tabela 1. Elementos do IO Data Bus

\begin{tabular}{|l|l|}
\hline \multicolumn{1}{|c|}{ Elemento } & \multicolumn{1}{c|}{ Característica } \\
\hline Barramento & Próprio sistema IO Data Bus. \\
\hline Sistemas de Informação (SI) & $\begin{array}{l}\text { Sistemas e Bancos de Dados governamentais } \\
\text { que estarão conectados ao barramento. }\end{array}$ \\
\hline Módulo Central & Gerência segurança, carimbos de tempo e logs. \\
\hline Módulos Clientes & Módulos escalávis que recebem requisições. \\
\hline Módulos Servidores & $\begin{array}{l}\text { Módulos escaláveis que recebem as requisições } \\
\text { dos módulos servidores e encaminham aos SIs } \\
\text { de destino. }\end{array}$ \\
\hline
\end{tabular}

Antes do atendimento da solicitação, o SI do órgão X busca algumas informações com o Y que por sua vez pede algumas informações complementares para um órgão Z. Então, para isso, Y faz a requisição para um Módulo Cliente que busca o Módulo Servidor, esse entrega a requisição ao Órgão Z. O Módulo Central é responsável por gerir toda a 
parte de segurança, verificando a conformidade de certificados de autoridades e também sendo composta por um serviço de carimbo de tempo ou timestamp.

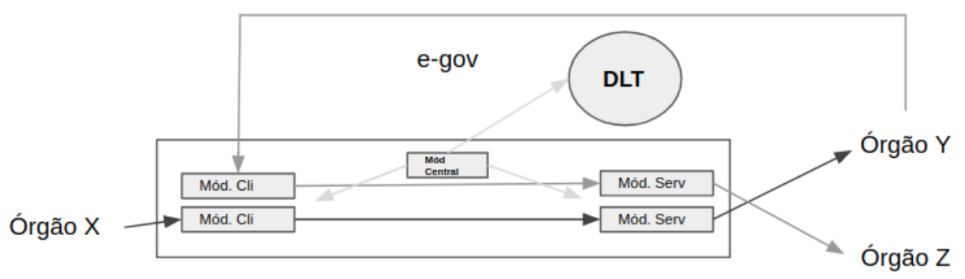

Figura 3. Estrutura básica do IO Data Bus no e-gov.

Todas as evidências que necessitam de registros são enviados do módulo central para uma DLT, devido a característica de imutabilidade dessa tecnologia, as DLTs vem se firmando cada vez mais ao longo dos anos. De acordo com [Tapscott and Tapscott 2016], essa tecnologia de Livro-Razão Distribuído, também conhecida como blockchain, tem o poder para mudar desde o meio de operação do comércio, até mesmo levar transformação à economia em escala global. De acordo com [Isaja and Soldatos 2018], um livro-razão distribuído é um sistema de dados que são replicados e armazenados em sincronia entre múltiplos nós de uma rede, onde todos os nós são pares da rede e não há um nó master que possui a cópia do livro-razão. Referenciando sua pretensão tecnológica inicial, em [Nakamoto 2008], foi apresentada a tecnologia Blockchain como uma arquitetura segura que desse suporte a proposta da cripto-moeda Bitcoin. O termo Blockchain sugere uma cadeia de blocos, onde cada bloco pode armazenar um conjunto de dados e transações.

\section{Planejamento Experimental}

A simulação de uma situação cotidiana de Governo Eletrônico foi desenvolvida em linguagem Python. Esse tecnologia proporciona uma melhor modularização e rapidez, por se tratar de uma linguagem leve e adaptável. Executamos uma implantação real do $X$ Road em uma máquina virtual e na máquina host foi disponibilizado o nosso protótipo de e-gov. Para embasar a simulação, utilizamos como exemplo de serviço público digital o pagamento do auxílio emergencial, pelo Governo Federal, simulamos através da nossa implementação diferentes módulos e bases de dados, como da Receita Federal, INSS e Caixa Econômica Federal, por exemplo. Todas as simulações foram feitas utilizando dados fictícios.

\subsection{Prova de Conceito}

A utilização de um barramento de troca de dados se deu a partir da instalação do $X$ Road que dará suporte à nossa prova de conceito. Paralelamente foi implementado um webservice que simula a validação de dados em deferentes módulos, que representarão as entidades governamentais citadas anteriormente. Cada um desses órgãos presentes no barramento tem como objetivo a validação de dados para a aprovação ou não da solicitação de auxílio emergencial pelo cidadão. O código-fonte do nosso ecossistema de simulação está disponível no endereço https://github.com/iodatabus/auxilioemergencial-com-xroad.git

A Figura 4 ilustra o fluxograma dos requisitos necessários para o pagamento do auxílio emergencial na nossa prova de conceito, para chegar a tais passos abstraímos 
as etapas realizadas, nas situações reais, pela Caixa Econômica Federal e DATAPREV para o processamento das requisições do pagamento do auxílio emergencial. Será utilizado como modelo de identificação única e digital o DNI ou Documento Nacional de Identificação, esse documento surgiu como uma proposta para unificação da maioria dos documentos oficiais dos brasileiros. Através do decreto $\mathrm{N}^{\circ} 9.278$ de 5 fevereiro de 2018, o DNI chegou a ser anunciado pelo governo federal e uma das suas características era de que a centralidade das informações seriam de responsabilidade do TSE (Tribunal Superior Eleitoral), dessa forma também utilizaremos uma abstração dessa documentação para centralização dos documentos [Federal 2018].

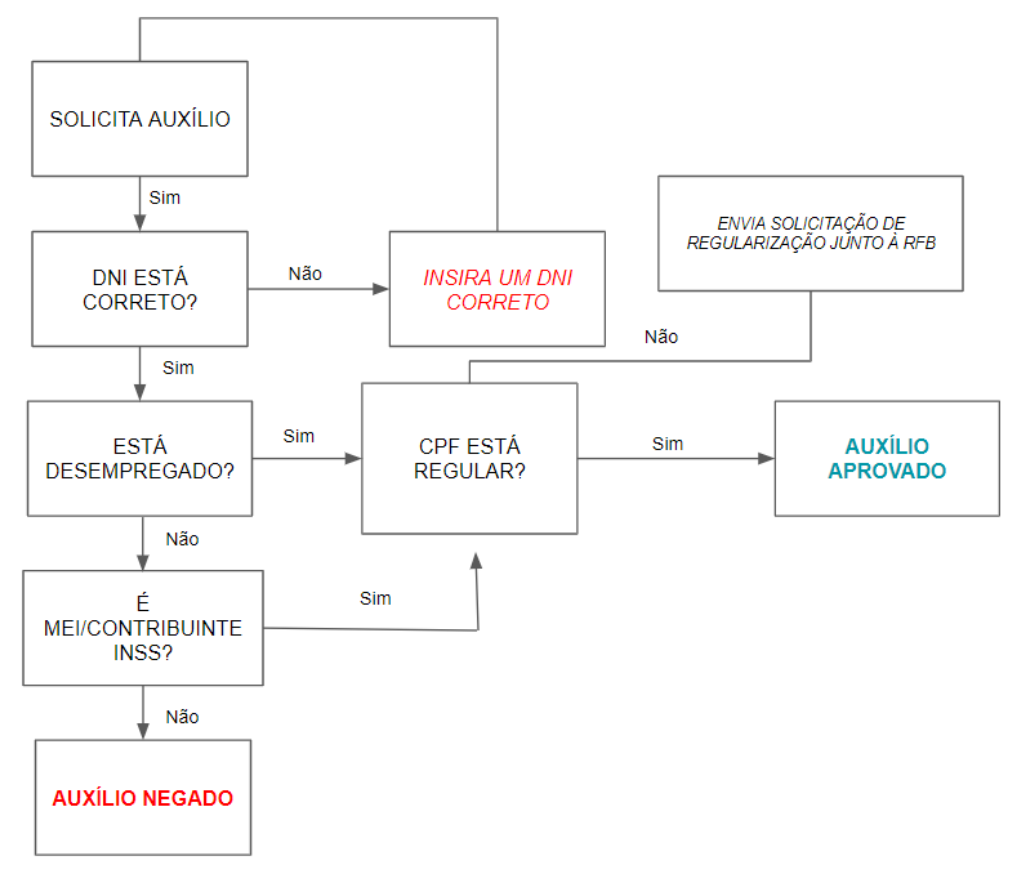

Figura 4. Fluxograma da Solicitação do Auxílio Emergencial na Prova de Conceito

Na Figura 5 está ilustrado o processo o fluxo da interação entre componentes presentes no nosso caso de uso, que é a simulação do processamento das solicitações de auxílio emergencial utilizando uma camada de troca de dados. Como é possível observar, implementamos um script que simulou o aplicativo real CaixaTem, onde esse recebe o conjunto de dados da solicitação (Nome Completo, Número do DNI, Data de Nascimento), esses dados são encaminhados para o $X$-Road, por meio de requisições REST/JSON, que trata os dados e faz todo o processo descrito na arquitetura da nossa proposta de barramento (armazenamento de logs, divisão em módulos, segurança criptográfica entre as trocas de requisições, etc). Com isso, o barramento encaminha esses dados para uma API, que faz a distribuição desses dados entre os diferentes módulos implementados, cada módulo possui uma base de dados fictícia para a validação dos dados. Após cada validação, seguindo o fluxo anteriormente demonstrado na Figura 4, o script do processamento retorna a mensagem de auxílio aprovado ou negado de acordo com as condicionais implementadas no projeto. 


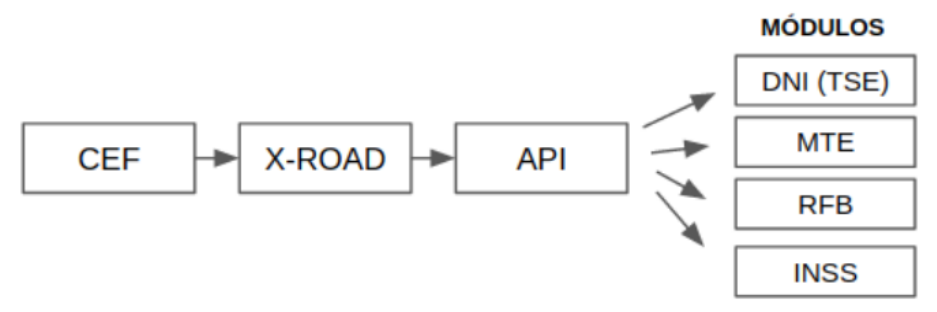

Figura 5. Fluxo da Interação entre os Componentes do Caso de Uso

Atualmente há uma grande centralização dos dados e pouca automatização tecnológica visando a validação dos dados para o processamento dos dados na interoperabilidade das entidades envolvidas de alguma forma com a concessão do auxílio.

De acordo com [DATAPREV 2021], esse fluxo, ilustrado na Figura 6, realiza o processamento real do Auxílio Emergencial. Como pode ser visto, a DATAPREV recebe a solicitação da Caixa, depois cruza os dados na base do Cadastro Nacional de Informações Sociais (CNIS), esse banco de dados possui mais de 33 bilhões de registros, após esse cruzamento é enviada ao Ministério da Cidadania as indicações de famílias elegíveis ao auxílio , fazendo assim a homologação e devolvendo para a DATAPREV, depois de todo esse processo é que a DATAPREV gera os arquivos homologados e os envia à Caixa Econômica Federal.

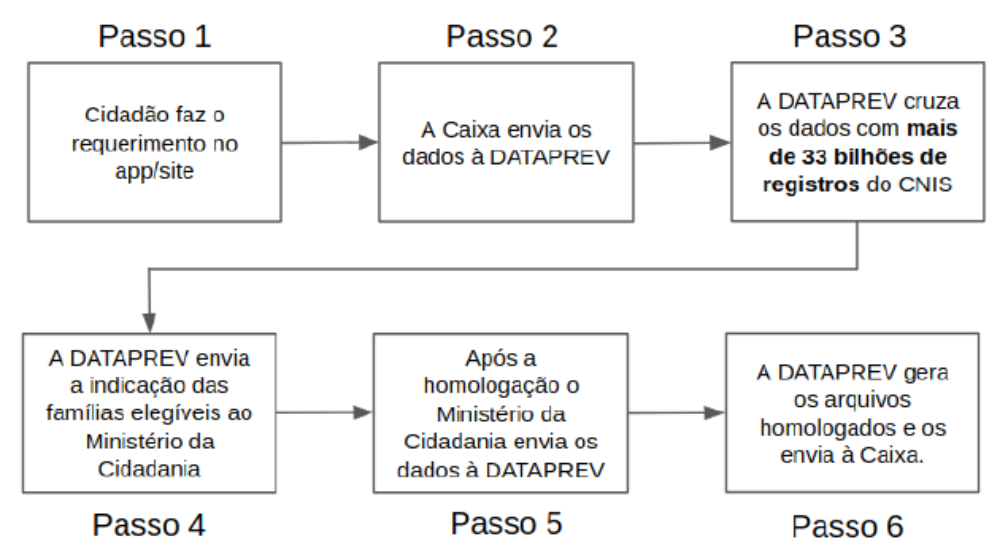

Figura 6. Fluxo Real da Solicitação do Auxílio Emergencial

\section{Resultados e Análise}

Visando experimentar uma maior interoperabilidade entre as entidades responsáveis pela validação do auxílio e com a nossa prototipação funcional e operante, realizamos uma série de testes para validar a prova de conceito proposta. Como citado anteriormente, temos um conjunto de dados fictícios em cada módulo dos órgãos no barramento. Com isso, os pedidos irão se basear nesses dados para o fornecimento do auxílio.

A Tabela 2 traz os cidadãos que utilizaremos no nosso experimento para a solicitação do auxílio. A primeira consulta será feita à base de dados do DNI, presente no TSE, então nosso script de solicitação irá enviar a requisição até ela através do X-Road. 
Tabela 2. Parte do Banco de Dados do módulo DNI (TSE)

\begin{tabular}{|l|l|l|l|l|}
\hline DNI & Nome Completo & Data de Nascimento & $C P F$ & $C T P S$ \\
\hline 123321123 & Joao Silva & $12 / 12 / 1955$ & 648812677 & 78941548484485 \\
\hline 123325553 & Maria Sampaio & $12 / 12 / 1955$ & 648812677 & 78941548484485 \\
\hline 123325669 & Joana Marques & $05 / 02 / 1985$ & 648812455 & 78941548486688 \\
\hline 123325784 & Alvaro Pereira & $04 / 11 / 1990$ & 648810000 & 78941548480078 \\
\hline
\end{tabular}

Na Figura 7 ilustramos a execução do Sistema de Informação de solicitação que implementamos. A requisição é enviada ao barramento, o SI provedor, a alusão à Caixa Econômica Federal, busca validar as informações em outras bases dados. No nosso resultado, a nossa cidadã fictícia Maria Sampaio teve seu benefício autorizado porque atendia todos os pré-requisitos necessários para a obtenção do auxílio (quando nosso script . Já o cidadão João da Silva teve o auxílio negado por não atender a um dos requisitos alguma das bases de dados verificadas através do barramento. Todo o output e resultados dos nossos testes, incluindo do cidadãos Joana Marques e Alvaro Pereira, estão presentes no repositório do projeto.

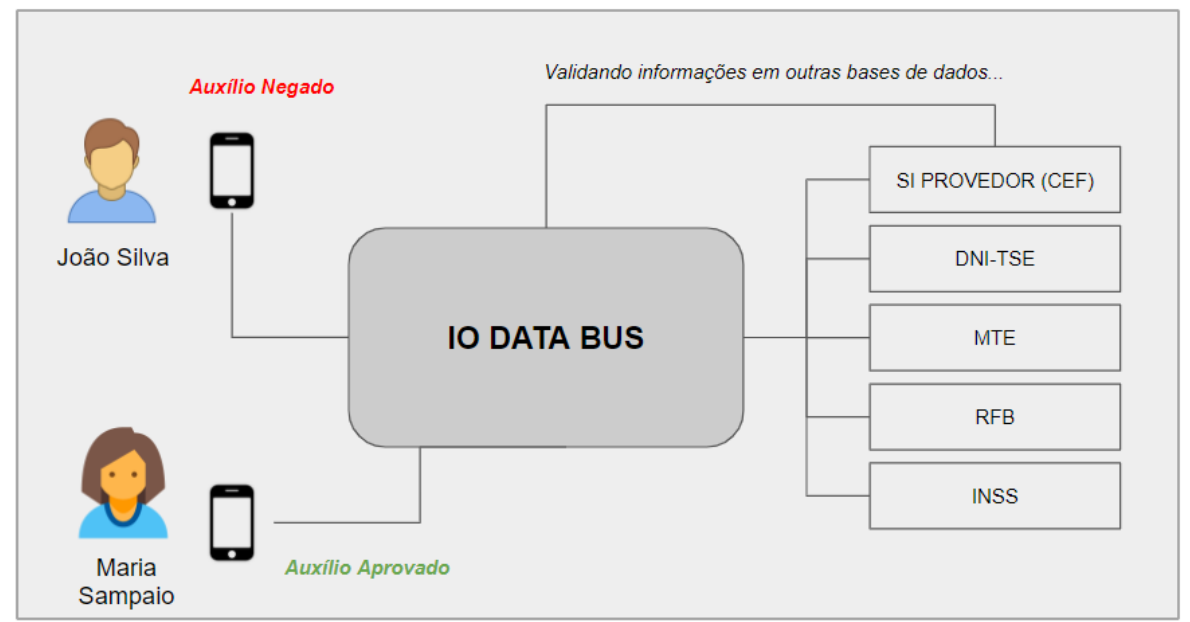

Figura 7. Executando a Solicitação do Auxílio no Barramento

De acordo com [Brasil 2020], somente entre abril e junho de 2020, 107 milhões de pessoas haviam solicitado o Auxílio Emergencial através do aplicativo e site fornecidos pela Caixa Econômica Federal, neste época o governo fechou um acordo com a Caixa Econômica e DATAPREV para analisar em até 20 dias as solicitações de auxílio [UOL 2020]. Para simular um possível teste de stress na nossa prova de conceito, implementamos testes de solicitações de auxílio no nosso ambiente com o X-Road. O módulo de teste de carga foi chamado de auxilio-egov-stress e também está disponível no nosso repositório.

Ainda segundo [Brasil 2020], no início do período citado anteriormente, houve quase 100 mil solicitações nas primeiras 24 horas. Com isso, utilizamos essa referência para simular o teste de stress no nosso ambiente, enviando 100 mil requisições REST ao 
barramento com diferentes cenários randômicos previamente planejados e inseridos nas diferentes bases de dados em que o barramento faz a conexão. Nesse contexto, a Figura 8 ilustra um gráfico comparativo entre máximo de dias que a resposta da solicitação do auxílio leva na situação real e na nossa proposta de barramento, levando em consideração as 100 mil requisições.

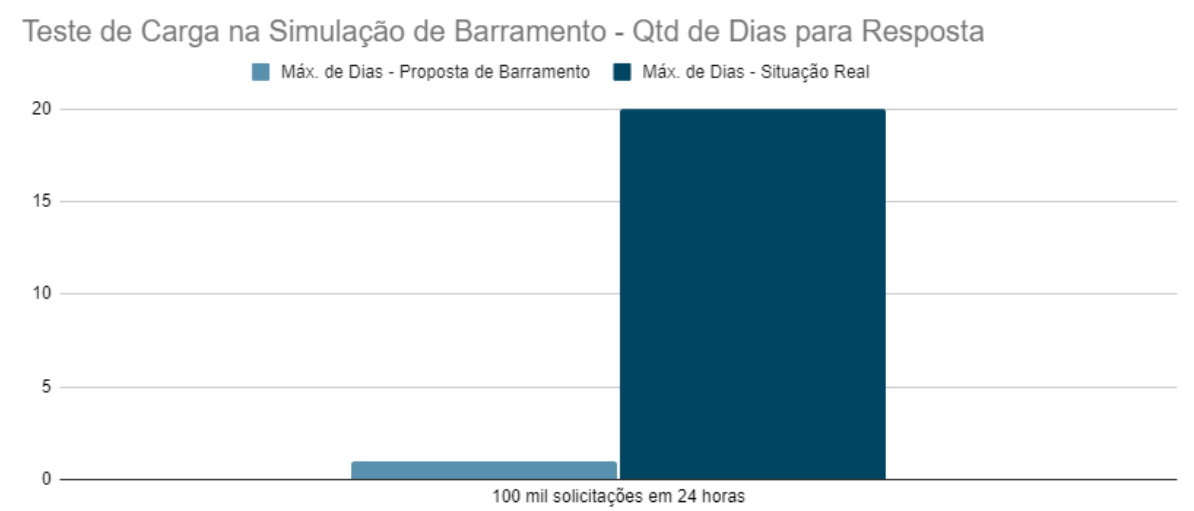

Figura 8. Resultado do Teste de Carga no Cenário de Prova de Conceito

\section{Conclusão e Trabalhos Futuros}

O presente trabalho propôs um barramento de troca de dados para ser aplicado em contextos de Governos Eletrônicos. Como prova de conceito foi utilizada a camada de troca de dados X-Road como um barramento no ecossistema de simulação da abstração do pagamento do Auxílio Emergencial do Governo Federal. Um dos aspectos positivos foi a percepção de uma alta velocidade na entrega da validação de dados quando há necessidade de uma boa interoperabilidade entre diferentes entidades governamentais.

Com a utilização de um barramento de troca de dados a simulação da abstração do pagamento do auxílio ocorreu de maneira rápida e automática, pois os diferentes Sistemas de Informação e Bancos de Dados fictícios, necessários para validação foram acessados em tempo real em diferentes simulações de órgãos, evitando assim uma grande concentração de dados em pontos únicos de falhas que podem ocasionar possíveis gargalos e indisponibilidade na nossa simulação. Como trabalhos futuros utilizaremos mais exemplos de casos de uso para validar nossas provas de conceito em situações de $e$-gov e serão focados os diferentes segmentos de interconexão do $e P I N G$ que ainda não foram utilizados nos casos de uso, além de uma maior utilização dos benefícios de imutabilidade e segurança de DLTs, fornecendo uma maior confiabilidade em evidências de transações dos dados que trafegam no barramento.

\section{Referências}

Brasil, A. (2020). Agência brasil - ebc. https://agenciabrasil.ebc.com.br/economia/noticia/202006/em-dois-meses-107-milhoes-de-brasileiros-pediram-auxilio-emergencial.

Brasileiro, G. (2018). e-ping padrões de interoperabilidade de governo eletrônico. Comitê Executivo de Governo Eletrônico, May.

Brizzi Neto, J. (2007). O supersimples e a micro e pequena empresa: aspectos sociais e jurídicos. 
da Purificação, C. S. (2019). A digitalização dos serviços públicos de atendimento no âmbito do governo federal: Um olhar para as mudanças na forma de prestação desses serviços após a adesão dos instrumentos digitais.

DATAPREV (2021). Portal da dataprev, https://portal2.dataprev.gov.br/.

Digital, G. (2020). Governo digital, https://www.gov.br/governodigital/pt-br.

dos Santos, E. M. (2011). A adoção da arquitetura e-ping: Um estudo de caso na fiocruz/bahia.

Federal, G. (2018). Decreto $\mathrm{n}^{\circ}$ 9.278, de 5 de fevereiro de 2018, https://www2.camara.leg.br/legin/fed/decret/2018/decreto-9278-5-fevereiro-2018786143-norma-pe.html.

Framework, I. (2004). European interoperability framework for pan-european egovernment services.

Isaja, M. and Soldatos, J. (2018). Distributed ledger technology for decentralization of manufacturing processes. In 2018 IEEE Industrial Cyber-Physical Systems (ICPS), pages 696-701. IEEE.

Jaeger, P. T. and Thompson, K. M. (2003). E-government around the world: Lessons, challenges, and future directions. Government information quarterly, 20(4):389-394.

Kalja, A. (2011). The first ten years of x-road. Estonian Information Society Yearbook, 2012:78-80.

Nakamoto, S. (2008). Bitcoin: A peer-to-peer electronic cash system. Technical report, Manubot.

Nielsen, M. M. (2017). egovernance and online service delivery in estonia. In Proceedings of the 18th Annual International Conference on Digital Government Research, pages 300-309.

NIIS (2020). X-road architecture, https://bitbucket.niis.org/projects/x-road/repos/xroad/browse/doc/architecture/arc-g_X-road_arhitecture.md/.

Saputro, R., Pappel, I., Vainsalu, H., Lips, S., and Draheim, D. (2020). Prerequisites for the adoption of the x-road interoperability and data exchange framework: A comparative study. In 2020 Seventh International Conference on eDemocracy \& eGovernment (ICEDEG), pages 216-222. IEEE.

Srivastava, S. C. and Teo, T. S. (2004). A framework for electronic government: evolution, enablers and resource drainers. In Proceedings of the Eighth Pacific Asia Conference on Information Systems.

Tapscott, D. and Tapscott, A. (2016). Blockchain revolution: how the technology behind bitcoin is changing money, business, and the world. Penguin.

UOL (2020). Auxílio: Governo terá prazo máximo de 20 dias corridos para analisar pedido, https://economia.uol.com.br/noticias/redacao/2020/06/01/auxilio-emergencialprazo-20-dias-analise-pedidos.htm. 